\title{
La structure algébrique de la représentation et le problème de la symétrie pour une classe de fonctionelles de Wightman
}

\author{
A. N. VASSILEV \\ l'Université de Leningrade, USSR \\ Reçu le 6 Fevrier 1969
}

\begin{abstract}
The special set $Q$ of Wightman's functionals is considered. All the pure functionals that belong to $Q$ have the strong spectral property and the single vacuum. All other functionals from $Q$ may be represented by integrals over (weak) compact sets of pure functionals from $Q$. The Borchers structure theorem is proved for this set. The conditions of the unitary equivalence of the representations and their similarity are given. These results are applied for the analysis of the symmetry problem. In particular we formulate the necessary and sufficient condition for the given group of automorphisms to be unitarily implemented.
\end{abstract}

\section{Description de la classe $Q$}

Soit $A$ la * algèbre de Borchers [2] constituée par des «chaînes» finies:

$$
a \equiv\left(a_{0}, a_{1}(x), a_{2}\left(x_{1}, x_{2}\right), \ldots\right)
$$

où $a_{0}$ - un nombre complexe, $a_{n}\left(x_{1} \ldots x_{n}\right)$ appartient à l'espace de Schwartz $S_{4 n}$. L'algèbre $A$ munie de la topologie limite inductive stricte de la somme directe des $S_{4 n}$ est un espace topologique nucléaire [3].

Soit $w$ une fonctionelle de Wightman, c'est à dire une fonctionelle (une forme linéaire) continue positive sur $A$, invariante par le groupe de PoIncaré (P्P-invariante) $[1,2]$ et satisfaisant à la condition spectrale $[1,2]$. Désignons par $K_{0}$ le cône (convexe, saillant, faiblement fermé) de ces fonctionelles, par $K$ - le cône (convexe, saillant, faiblement fermé) de toutes les fonctionelles positives.

Nous n'exigeons pas la localité et ne l'utilisons pas sauf mention explicite du contraire. Dans toute la suite de cet article nous supposons $w$ normée à l'unité $(w(1)=1)$.

Désignons par $R_{w}$ la représentation construite à partire d'une $w \in K_{0}$ par le procédé du théorème de la reconstruction $[1,2]$, par $r_{w}(a): a \in A$ - ses éléments, par $H_{w}$ - l'espace hilbertien de la représentation, par $\psi_{w}(1)$ - le vecteur cyclique. Les opérateurs de $R_{w}$ sont définis sur

6 Commun. math. Phys., Vol. 13

$$
L_{w} \equiv\left\{\psi_{w}(a) \equiv r_{w}(a) \psi_{w}(1): a \in A\right\}
$$


qui est dense dans $H_{w}$ par construction (dans la suite nous appellerons cette propriété cyclicité de $R_{w}$ ). On a:

$$
w(a)=\left\langle\psi_{w}(1), r_{w}(a) \psi_{w}(1)\right\rangle=\left\langle\psi_{w}(1), \psi_{w}(a)\right\rangle .
$$

Désignons par $U_{w}(\lambda, \Lambda)$ la représentation unitaire du groupe de Poincaré dans $H_{w}$ :

$$
U_{w}(\lambda, \Lambda) \psi_{w}(a)=\psi_{w}([\lambda, \Lambda] a)
$$

où $\lambda \in R^{4}$ est un vecteur de la translation, $\Lambda$ - un élément du groupe de LoRENTz homogène, $[\lambda, \Lambda]$ - la représentation canonique du groupe de Poincaré par les *automorphismes de l'algèbre $A[1,2]$.

Désignons par $H_{w}^{0}$ le sous-espace de vide de l'espace $H_{w}$ c'est à dire l'ensemble de vecteurs invariants par $U_{w}(\lambda, 1)$ :

$$
x \in H_{w}^{0} \Leftrightarrow U_{w}(\lambda, 1) x=x \forall \lambda \in R^{4} .
$$

Sous les hypothèses traditionnelles y compris la localité, Borchers [2] a démontré que $H_{w}^{0}$ est $\mathscr{P}$-invariant:

$$
U_{w}(\lambda, \Lambda) x=x \forall x \in H_{w}^{0} .
$$

La localité n'étant pas exigée, nous n'avons pas le droit d'exploiter cette assertion.

Définition. Disons qu'une fonctionelle $w \in K_{0}$ appartient à la classe $Q_{0}$ si elle:

1. est un point extrémal de $K_{0}$,

2. satisfait à la condition spectrale forte [2],

3. a le vide non dégénéré (le sous-espace de vide $H_{w}^{0}$ est de dimension 1 ).

Un point extrémal dans $K_{0}$ peut ne pas l'être dans $K$. Démontrons quand même.

Proposition 1. Supposons qu'une $w \in K_{0}$ a la décomposition:

$$
\begin{aligned}
& w=\varrho_{1} w_{1}+\varrho_{2} w_{2} \quad \varrho_{1,2}>0 \\
& \varrho_{1}+\varrho_{2}=1 \quad w_{1} \neq w_{2} \quad w_{1,2} \in K
\end{aligned}
$$

Alors les fonctionelles $w_{1,2}$ sont nécessairement invariantes par le groupe des translations et satisfont à la condition spectrale. Si le sous-espace de vide $H_{w}^{0}$ est $\mathscr{P}$-invariant, les fonctionelles $w_{1,2}$ sont aussi $\mathscr{P}_{\text {-invariantes. }}$

Si $w$ satisfait à la condition spectrale forte il en est de même de $w_{1,2}$. Si $w$ est locale, il en est de même de $w_{1,2}$.

Lemme 1. Soient $w \in K_{0}, R_{w}$ la représentation correspondante dans $H_{w}, x-$ un vecteur dans $H_{w}$. Si la fonctionelle:

est symétrique:

$$
w_{x}(a) \equiv\left\langle x, \psi_{w}(a)\right\rangle
$$

$$
w_{x}\left(a^{+}\right)=w_{x}(a)^{*}
$$

le vecteur $x$ est un vide dans $H_{w}$. 
En effet, par hypothèse :

d'où :

$$
\left\langle x, \psi_{w}\left(a^{+}\right)\right\rangle=\left\langle\psi_{w}(a), x\right\rangle \forall a \in A
$$

$$
\left\langle x, \psi_{w}\left([\lambda, 1] a^{+}\right)\right\rangle=\left\langle\psi_{w}([\lambda, 1] a), x\right\rangle
$$

pour tout $a \in A$ et toute translation $\lambda \in R^{4}$. Ecrivons:

$$
\int e^{i p \lambda} d\left\langle x, E_{p} \psi_{w}\left(a^{+}\right)\right\rangle=\int e^{-i p \lambda} d\left\langle E_{p} \psi_{w}(a), x\right\rangle
$$

où $E_{p}$ est une mesure à valeurs projecteurs:

$$
U_{w}(\lambda, 1)=\int e^{i p \lambda} d E_{p}
$$

dont le support est contenu dans le cône futur:

$$
\bar{L}_{+} \equiv\left\{p \in R^{4}: p^{2} \equiv p_{0}^{2}-\boldsymbol{p}^{2} \geqq 0, p_{0} \geqq 0\right\}
$$

en vertu de la condition spectrale. Par suite les deux parties de l'équation ci-dessus étant considérées comme des fonctions de $\lambda_{0}$ complexe (pour un $\lambda \in R^{3}$ fixé) sont valeurs au bord des fonctions holomorphes et bornées dans les régions $\operatorname{Im} \lambda_{0}>0, \operatorname{Im} \lambda_{0}<0$ respectivement. Etant égales sur l'axe réel ces fonctions constituent une fonction holomorphe et bornée dans tout le plan de $\lambda_{0}$ complexe, donc cette fonction est constante, d'où la conclusion.

Passons maintenant à la démonstration de la proposition 1. Soit done une $w \in K_{0}$ ayant la décomposition:

$$
w=\varrho_{1} w_{1}+\varrho_{2} w_{2} \varrho_{1,2}>0 \varrho_{1}+\varrho_{2}=1 w_{1} \neq w_{2} w_{1,2} \in K
$$

Démontrons que $w_{1,2}$ sont invariantes par des translations.

Pour le faire, réalisons $R_{w}$ et $R_{w_{1,2}}$ comme d'habitude et considérons les applications linéaires $V_{i} i=1,2$ de $L_{w} \subset H_{w}$ sur $L_{w_{i}} \subset H_{w_{i}}$ respectivement:

$$
V_{i} \psi_{w}(a)=\psi_{w_{i}}(a), \quad i=1,2 .
$$

Ces applications sont bornées:

Ecrivons:

$$
\left\|V_{i} \psi_{w}(a)\right\|^{2}=\left\|\psi_{w_{i}}(a)\right\|^{2}=w_{i}\left(a^{+} a\right) \leqq \frac{1}{\varrho_{i}} w\left(a^{+} a\right)=\frac{1}{\varrho_{i}}\left\|\psi_{w}(a)\right\|^{2} .
$$

$$
w_{i}(a)=\left\langle\psi_{v_{i}}(1), \psi_{w_{i}}(a)\right\rangle=\left\langle V_{i}^{+} \psi_{w_{i}}(1), \psi_{w}(a)\right\rangle .
$$

Les fonctionelles $w_{1,2}$ sont positives, done symétriques et en vertu du lemme 1 les vecteurs $V_{i}^{+} \psi_{w_{i}}(1)$ appartiennent à $H_{w}^{0}$. Il en résulte que $w_{1,2}$ sont invariantes par des translations et si en outre le sous-espace de vide $H_{w}^{0}$ est $\mathscr{P}$-invariant, il en est de même de $w_{1,2}$.

Avant de continuer la démonstration formulons la condition spectrale etc. d'une façon compacte.

Soient $a_{n}\left(x_{1} \ldots x_{n}\right)$ une fonction de l'espace de Schwartz $S_{4 n}$ $\tilde{a}_{n}\left(p_{1} \ldots p_{n}\right)$ - sa transformée de FouRIER. Désignons par $\operatorname{Supp}_{p} a_{n}$ l'ensemble de points $p \in R^{4}$ tels que la fonction de $n-1$ variables 6* 
$p_{1} \ldots p_{n-1}: \tilde{a}_{n}\left(p_{1} \ldots p_{n-1}, p-\sum_{i=1}^{n-1} p_{i}\right)$ n'est pas nulle. Désignons:

$$
\operatorname{Supp}_{p} a \equiv \bigcup_{n} \operatorname{Supp}_{p} a_{n}
$$

en entendant sous $\operatorname{Supp}_{p} a_{0}$ le point $p=0$ si $a_{0} \neq 0$ et l'ensemble vide dans le cas contraire.

Alors la condition spectrale s'écrit [2]:

$$
\psi_{w}(a)=0
$$

pour tout $a \in A$ tel que $\operatorname{Supp}_{p} a \cap \bar{L}_{+}=\emptyset$.

La condition spectrale forte s'écrit:

$$
\psi_{w}(a)=0
$$

pour tout $a \in A$ tel que $\operatorname{Supp}_{p} a \cap\left\{p=0 \cup V_{\mu}\right\}=\emptyset$ où $V_{\mu}$ est l'hyperboloîde:

$$
V_{\mu} \equiv\left\{p \in R^{4}: p^{2} \geqq \mu^{2}>0, p_{0}>0\right\}
$$

contenant le spectre continu de l'opérateur d'énergie-impulsion.

La condition de la localité s'écrit: $\psi_{w}(a)=0$ pour tout $a \in I_{c}$ où $I_{c}$ (la notation de l'article [2]) est l'idéal bilatére engendré par les éléments de la forme:

$$
\left(0,0, a\left(x_{1}\right) b\left(x_{2}\right)-b\left(x_{1}\right) a\left(x_{2}\right), 0,0, \ldots\right)
$$

où $a, b$ sont des fonctions à support compact telles que la séparation entre $\operatorname{Supp}_{x} a$ et $\operatorname{Supp}_{x} b$ est de genre espace (pour les détails voir [2]).

Pour achever la démonstration de la proposition 1 il suffit de se servir de ces définitions et de l'inégalité:

$$
\left\|\psi_{w_{i}}(a)\right\|^{2} \leqq \frac{1}{\varrho_{i}}\left\|\psi_{w}(a)\right\|^{2}
$$

Corollaire 1. Toute fonctionelle locale extrémale dans le cône des fonctionelles locales est extrémale dans le cône de toutes les fonctionelles positives.

Corollaire 2. Toute $w \in Q_{0}$ est extrémale dans $K$.

Dans ce cas il n'y a pas de question de l'invariance relativiste de $H_{w}^{0}$ car il est engendré par un vecteur $\psi_{w}(1)$ (condition 3 ) qui est $\mathscr{P}$-invariant par définition.

Désignons par $T$ le groupe de tous les * automorphismes de l'algèbre $A$ continus pour sa topologie, par $T^{0}$ le sousgroupe des * automorphismes permutables avec les * automorphismes du groupe de PoIncaré.

Proposition 2. Soient $w \in Q_{0}, \tau \in T^{0}$. Définissons;

$$
w_{\tau}(a) \equiv w(\tau a) \text {. }
$$

La fonctionelle $w_{\tau}$ apportient $\grave{a} Q_{0}$.

Il est évident que $w_{\tau}$ est positive et $\mathscr{P}$-invariante. Elle est extrémale dans $K$, sinon $w$ serait aussi décomposable:

$$
w_{\tau}=\varrho_{1} w_{1}+\varrho_{2} w_{2} \Rightarrow w=\varrho_{1}\left(w_{1}\right)_{\tau-1}+\varrho_{2}\left(w_{2}\right)_{\tau-1} .
$$


Démontrons que $w_{\tau}$ satisfait à la condition spectrale forte et à celle d'unicité de vide.

Soient $R_{w}, \mathrm{R}_{w_{\tau}}$ les représentations dans $H_{w}, H_{w_{\tau}}$ respectivement. Considérons l'application $V_{\tau}$ de $L_{w} \subset H_{w}$ sur $L_{w \tau} \subset H_{w \tau}$ :

$$
V_{\tau} \psi_{w}(\tau a)=\psi_{w_{\tau}}(a) \forall a \in A \text {. }
$$

Cette application est évidemment isométrique et peut être prolongée par continuité sur tout $H_{w}$. En utilisant les définitions et la condition $\tau \in T^{0}$, nous trouvons:

$$
U_{w_{\tau}}(\lambda, \Lambda)=V_{\tau} U_{w}(\lambda, \Lambda) V_{\tau}^{-1}
$$

Cela signifie que les représentations unitaires du groupe de PoINcaré dans $H_{w}$ et $H_{w \tau}$ sont unitairement équivalentes ce qui entraîne la coîncidence des spectres des opérateurs d'énergie-impulsion et de dimensions des sous-espaces de vide.

Soient $w \in K_{0}, R_{w}$ la représentation correspondante dans $H_{w}$. A la suite de RUELLE [4], nous appelons commutant de la représentation $R_{w}$ (désignons par $R_{w}^{\prime}$ ) l'ensemble de tous les opérateurs bornés $B$ dans $H_{w}$ tels que l'égalité :

$$
\left\langle\psi_{w}(a), B \psi_{w}(b c)\right\rangle=\left\langle\psi_{w}\left(b^{+} a\right), B \psi_{w}(c)\right\rangle
$$

est valide pour tous les $a, b, c \in A$.

Il est évident que $R_{w}^{\prime}$ contient avec deux opérateurs $B_{1,2}$ leurs combinaisons linéaires et leurs ajoints $B_{1,2}^{+}$.

Lemme 2. Soient $w \in K_{0}, R_{w}$ la représentation correspondante dans $H_{w}$, $B$ un opérateur du commutant $R_{w}^{\prime}$.

Le vecteur $B \psi_{w}(1)$ est un vide dans $H_{w}$.

Il suffit de le démontrer pour les opérateurs auto-ajoints de $R_{w}^{\prime}$. Soit donc un $B=B^{+} \in R_{w}^{\prime}$. La fonctionelle:

est symétrique:

$$
w_{B}(a) \equiv\left\langle\psi_{w}(1), B \psi_{w}(a)\right\rangle
$$

$$
w_{B}\left(a^{+}\right)=\left\langle\psi_{w}(1), B \psi_{w}\left(a^{+}\right)\right\rangle=\left\langle\psi_{w}(a), B \psi_{w}(1)\right\rangle=w_{B}(a)^{*}
$$

d'où la conclusion (lemme 1).

Notons qu'en démontrant les lemmes 1,2 nous avons suivi le raisonnement de Borchers ([2], théorème 4).

Proposition 3. Soit une $w \in Q_{0}$. Le commutant $R_{w}^{\prime}$ est trivial.

Cette assertion est bien connue [4]. Démonstration. Soit un $B \in R_{w}^{\prime}$. On a:

$$
\left\langle\psi_{w}(a), B \psi_{w}(b)\right\rangle=\left\langle\psi_{w}\left(b^{+} a\right), B \psi_{w}(1)\right\rangle .
$$

En vertu du lemme $2 B \psi_{w}(1)$ est un vide. Le vide étant unique par hypothèse $\left(w \in Q_{0}\right)$, il en résulte qu'il existe un nombre complexe $c$ tel que:

$$
\left\langle\psi_{w}(a), B \psi_{w}(b)\right\rangle=c\left\langle\psi_{w}\left(b^{+} a\right), \psi_{w}(1)\right\rangle=c\left\langle\psi_{w}(a), \psi_{w}(b)\right\rangle
$$

d'où $B=c$ à la suite de la cyclicité de $R_{w}$. 
Passons maintenant à la description de la classe $Q$. Soit $s \subset Q_{0}$ un ensemble de fonctionelles satisfaisant aux conditions suivantes:

4. $s$ est fermé et borné pour la topologie faible de l'espace des fonctionelles continues sur $A$,

5. toutes les fonctionelles de $s$ ont la masse minimale commune; de facon précise, il existe un $\mu>0$ tel que pour toute fonctionelle $w \in s$ le spectre continu de l'opérateur d'énergie-impulsion est contenu à l'intérieur de l'hyperboloîde $V_{\mu}$.

Etant fermé et borné, $s$ est compact pour la topologie faible [5].

Il est évident que tout ensemble fini de points de $Q_{0}$ satisfait à ces conditions. Plus loin nous donnerons un exemple de $s$ contenant un nombre infini de points de $Q_{0}$.

En vertu de la condition 4 les fonctions $f_{a}(w) \equiv w(a)(a \in A$ étant fixé, $w$ parcourt $s$ ) appartiennent à l'espace de BANACH $C_{0}(s)$ des fonctions continues (pour la topologie faible) bornées sur $s$.

Lemme 3. L'ensemble $F(s)$ des fonctions $f_{a}(w): a \in A$ est dense dans $C_{0}(s)$ pour la topologie uniforme.

Démontrons que $F(s)$ satisfait aux conditions du théorème de VeIterstrass-STONe [6] qui affirme que tout ensemble de fonctions continues bornées sur un espace compact $X$ algébriquement clos et séparent les points de $X$ est uniformément dense dans $C_{0}(X)$.

$F(s)$ sépare les points de $s$ car l'égalité $f_{a}\left(w_{1}\right)=f_{a}\left(w_{2}\right) \forall a \in A$ implique $w_{1}=w_{2}$. Ensuite:

$$
\lambda_{1} f_{a_{1}}(w)+\lambda_{2} f_{a_{2}}(w)=f_{\lambda_{1} a_{1}+\lambda_{2} a_{2}}(w) \in F(s), f_{a}(w)^{*}=f_{a^{+}}(w) \in F(s) .
$$

Il nous reste à dèmontrer que $F(s)$ contient tous les produits $f_{a}(w) f_{b}(w)$. Pour le faire nous exploiterons la condition spectrale forte.

Tout $a \in A$ peut être décomposé en somme:

$$
a=a^{(0)}+a^{(1)}+a^{(2)}, a^{(i)} \in A i=0,1,2
$$

de sorte que $\operatorname{Supp}_{p} a^{(0)}$ soit contenu dans un voisinage du point $p=0$, $\operatorname{Supp}_{p} a^{(1)}$-dans un voisinage de $V_{\mu}$ et

$$
\operatorname{Supp}_{p} a^{(2)} \cap\left\{p=0 \cup V_{\mu}\right\}=\emptyset .
$$

Alors $\psi_{w}\left(a^{(2)}\right)=0 \forall w \in s$ en vertu de la condition spectrale forte, $\psi_{w}\left(a^{(0)}\right)$ est un vide dans $H_{w}, \psi_{w}\left(a^{(1)}\right)$ est orthogonal au vide de $H_{w}$. De facon précise:

$$
\psi_{w}\left(a^{(0)}\right)=w\left(a^{(0)}\right) \psi_{w}(1)=w(a) \psi_{w}(1), \psi_{w}\left(a^{(1)}\right)=\psi_{w}(a)-w(a) \psi_{w}(1) .
$$

Soient $a, b$ deux éléments fixés de $A$. Le produit $a^{(0)} b^{(0)}$ appartient à $A$ et

$$
\begin{aligned}
f_{a^{(0)} b^{(0)}}(w) & =w\left(a^{(0)} b^{(0)}\right)=\left\langle\psi_{w}(1), r_{w}\left(a^{(0)}\right) \psi_{w}\left(b^{(0)}\right)\right\rangle \\
& =w(b)\left\langle\psi_{w}(1), r_{w}\left(a^{(0)}\right) \psi_{w}(1)\right\rangle=w(b) w(a)=f_{a}(w) f_{b}(w)
\end{aligned}
$$

d'où la conclusion. 
Soit $\mu$ une mesure positive sur $s$ [c'est à dire, la forme linéaire continue positive sur $\left.C_{0}(s)\right]$ de masse totale égale à $1(\mu(s)=1)$. L'intégrale:

$$
\int_{s} w(a) d \mu(w) \equiv \bar{w}(a)
$$

détermine une forme linéaire positive sur $A$. Démontrons sa continuité. Pour faire cela, passons à l'espace réel $A_{R} \equiv\left\{a \in A: a^{+}=a\right\} \bar{w}$ est alors une forme linéaire réelle sur $A_{R}$ dont nous devons démontrer la continuité.

$A_{R}$ est nucléaire, donc tonnelé. Pour les espaces tonnelés tout ensemble faiblement borné de fonctionelles est équicontinu [5], ce qui s'applique à l'ensemble $s$.

Soit $a_{n} \in A_{R}: n=1,2, \ldots$ une suite qui converge vers 0 pour la topologie de $A_{R}$. L'équicontinuité de $s$ permet d'affirmer que la suite des fonctions $f_{a_{n}}(w): n=1,2, \ldots$ converge vers 0 uniformément. Pour achever la démonstration il faut se rappeler que la fonctionelle $\bar{w}$ est continue par rapport à la convergence uniforme par construction.

Il est clair que $\bar{w}$ est $\mathscr{P}$-invariante et satisfait à la condition spectrale forte.

Définition. Nous dirons qu'une $w \in K_{0}$ appartient à la classe $Q$ si elle est de la forme $(*)$ où $s$ satisfait aux conditions 4,5.

Sans restreindre la généralité on peut supposer que $s$ coincide avec le support de la mesure $\mu$ (dans ce qui suit nous le supposerons).

En conclusion de ce paragraphe vérifions que la représentation (*) est unique en ce sens que l'égalité:

$$
\int_{s_{1}} w(a) d \mu_{1}(w)=\int_{s_{2}} w(a) d \mu_{2}(w) \quad \forall a \in A
$$

où $s_{i}=\operatorname{Supp} \mu_{i}$ satisfont aux conditions 4,5 entraîne:

$$
s_{1}=s_{2} \quad \mu_{1}=\mu_{2} .
$$

En effet l'ensemble $s \equiv s_{1} \cup s_{2}$ satisfait évidemment aux conditions 4,5 et les mesures $\mu_{1,2}$ peuvent être prolongées aux mesures $\tilde{\mu}_{1,2}$ sur $s$ dont les supports sont contenus dans $s_{1,2}$ respectivement. Plus précisement: pour toute fonction $f \in C_{0}(s)$ l'application $T_{i}$ de $C_{0}(s)$ à $C_{0}\left(s_{i}\right)$ :

$$
T_{i} f=\text { restriction de } f \grave{\mathrm{a}} s_{i}
$$

est bornée et par suite la forme linéaire $\tilde{\mu}_{i}$ sur $C_{0}(s)$ :

$$
\tilde{\mu}_{i}(f) \equiv \mu_{i}\left(T_{i} f\right)
$$

est continue pour la topologie uniforme de $C_{0}(s)$. L'égalité:

Supp $\tilde{\mu}_{i}$ dans $s=\operatorname{Supp} \mu_{i}$ dans $s_{i}=s_{i}$ est évidente.

L'ensemble $F(s)$ étant dense dans $C_{0}(s)$ (lemme 3), les mesures $\tilde{\mu}_{1,2}$ doivent être égales, d'où $s_{1}=s_{2}, \mu_{1}=\mu_{2}$. 


\section{Structure algébrique de la représentation pour les fonctionelles de la classe $\boldsymbol{Q}$}

Soient $\bar{w} \in Q, R_{w}$ la représentation dans $H_{w}$ construite à partir de $w \in s$ par le procédé du théorème de la reconstruction [1-2]. Soit

$$
H_{\bar{w}}=\int_{s} \oplus H_{w} d \mu(w)
$$

Par définition $H_{\bar{w}}$ contient toutes les fonctions à valeurs vecteurs:

$$
x \equiv\left\{[x]_{w} \in H_{w}: w \in s\right\}
$$

telles que la fonction numérique $\left\|[x]_{w}\right\|_{H_{w}}$ sur $s$ appartient à l'espace $L_{2}(\mu, s)$ des fonctions de carré $\mu$-intégrable sur $s$. Désignons:

$$
\begin{aligned}
\psi_{w}(1,1) & \equiv\left\{\psi_{w}(1): w \in s\right\} \\
\psi_{\bar{w}}(a, 1) & \equiv\left\{\psi_{w}(a): w \in s\right\} \\
L_{\bar{w}}(1) & \equiv\left\{\psi_{\bar{w}}(a, 1): a \in A\right\} .
\end{aligned}
$$

Evidemment $\psi_{\bar{w}}(a, 1) \in H_{\bar{w}}$ pour tout $a \in A$.

Définissons:

$$
R_{\bar{w}}=\int_{s} \oplus R_{w} \quad U_{\bar{w}}(\lambda, \Lambda)=\int_{s} \oplus U_{w}(\lambda, \Lambda) .
$$

Les opérateurs de $R_{\bar{w}}$ sont définis sur $L_{\bar{w}}(1)$ :

$$
r_{\bar{w}}(a) \psi_{\bar{w}}(b, 1)=\psi_{\bar{w}}(a b, 1) \quad \forall a, b \in A
$$

les opérateurs de $U_{\bar{w}}(\lambda, \Lambda)$ - sur tout $H_{\bar{w}}$.

Par construction le sous-espace de vide $H_{w}^{0}$ est $\mathscr{P}$-invariant et isomorphe à $L_{2}(\mu, s)$ :

$$
H_{\bar{w}}^{0}=\left\{\psi_{\bar{w}}(1, f): f \in L_{2}(\mu, s)\right\} \quad \text { où } \quad\left[\psi_{\bar{w}}(1, f)\right]_{w}=f(w) \psi_{w}(1)
$$

( $[x]_{w}$ est le composant d'un $x \in H_{\bar{w}}$ dans $\left.H_{w}\right)$. On a :

$$
\bar{w}(a)=\left\langle\psi_{\bar{w}}(1,1), r_{\bar{w}}(a) \psi_{\bar{w}}(1,1)\right\rangle .
$$

Pour démontrer que cette représentation coincide (à une équivalence unitaire près) avec celle qu'on obtient à partir de $\bar{w}$ par le procédé du théorème de la reconstruction, il nous faut vérifier la cyclicité de cette représentation. Autrement dit nous devons démontrer que $L_{\bar{w}}(1)$ est dense dans $H_{\bar{w}}$.

Proposition 4. $R_{\bar{w}}$ est cyclique.

Soit une $f \in L_{2}(\mu, s)$. Désignons par:

$$
\begin{aligned}
& \psi_{\bar{w}}(a, f) \equiv\left\{f(w) \psi_{w}(a): w \in s\right\}, \\
& L_{\bar{w}}(f) \equiv\left\{\psi_{\bar{w}}(a, f): a \in A\right\} \quad L_{\bar{w}} \equiv \bigcup_{f \in L_{2}(\mu, s)} L_{\bar{w}}(f) .
\end{aligned}
$$

Démontrons d'abord que $L_{\bar{w}}$ est dense dans $H_{\bar{w}}$. 
Supposons qu'il existe $0 \neq x \in H_{\bar{w}}$ tel que

$$
\left\langle x, \psi_{\bar{w}}(a, f)\right\rangle=\int_{s}\left\langle[x]_{w}, \psi_{w}(a)\right\rangle f(w) d \mu(w)=0
$$

pour tout $a \in A$ et toute $f \in L_{2}(\mu, s)$. Il en résulte que pour tout $a \in A$ la fonction numérique $\left\langle[x]_{w}, \psi_{w}(a)\right\rangle \in L_{2}(\mu, s)$ est nulle presque partout sur $s$.

Soit $a_{n}: n=1,2, \ldots$ un ensemble dense dans $A$ (il existe car $A$ est séparable [2]). Désignons par:

$$
e_{i} \equiv\left\{w \in s:\left\langle[x]_{w}, \psi_{w}\left(a_{i}\right)\right\rangle \neq 0\right\} \quad e \equiv \bigcup_{i=1}^{\infty} e_{i} .
$$

Nous avons démontré que tout ensemble $e_{i}$ est $\mu$-négligeable par suite leur réunion $e$ est aussi $\mu$ négligeable [7].

Désignons par $e^{\prime}$ le complémentaire de $e$ dans $s$. Soit une $v \in e^{\prime}$. On a :

$$
\left\langle[x]_{w}, \psi_{w}\left(a_{i}\right)\right\rangle=0 \quad \forall i=1,2, \ldots .
$$

Puisque l'ensemble $a_{i} i=1,2, \ldots$ est dense dans $A$ et l'application $a \rightarrow \psi_{w}(a)$ est continue l'ensemble $\psi_{w}\left(a_{i}\right) i=1,2, \ldots$ est dense dans $L_{w} \subset H_{w}$ et par suite, dans $H_{w}$, d'où $[x]_{w}=0 \forall w \in e^{\prime}$, donc presque partout. Il en résulte que $x$ est nul dans $H_{\bar{w}}$, ce qui contredit l'hypothèse.

Il nous reste à démontrer que $L_{\bar{w}}(1)$ est dense dans $L_{\bar{w}}$. Nous le ferons en exploitant la condition spectrale forte.

Soient $a \in A, f \in L_{2}(\mu, s)$ fixés. Désignons :

$$
u(a) \equiv \operatorname{Sup}_{w \in s} w\left(a^{+} a\right) \text {. }
$$

L'ensemble $F(s)$ étant uniformement dense dans $C_{0}(s)$ est dense dans $L_{2}(\mu, s)$ pour sa topologie. Il existe donc un $b \in A$ tel que:

$$
\int_{s}\left|f_{b}(w)-f(w)\right|^{2} d \mu(w)<\frac{\varepsilon}{u(a)}
$$

( $\varepsilon>0$ est arbitraire). Décomposons $b$ en somme $b^{(0)}+b^{(1)}+b^{(2)}$ (voir la démonstration du lemme 3). On a:

$$
f_{b}(w)=w(b)=w\left(b^{(0)}\right)=f_{b^{(0)}}(w) .
$$

Considérons le vecteur $\psi_{\bar{w}}\left(a b^{(0)}, 1\right) \in L_{\bar{w}}(1)$. On a :

$$
\begin{aligned}
& \left\|\psi_{\bar{w}}\left(a b^{(0)}, 1\right)-\psi_{w}(a, f)\right\|^{2}=\int_{s}\left\|\psi_{w}\left(a b^{(0)}\right)-f(w) \psi_{w}(a)\right\|^{2} d \mu(w) \\
& =\int_{\delta}\left\|\left(f_{b}(w)-f(w)\right) \psi_{w}(a)\right\|^{2} d \mu(w)=\int_{s} w\left(a^{+} a\right)\left|f_{b}(w)-f(w)\right|^{2} d \mu(w)<\varepsilon
\end{aligned}
$$

d'où la conclusion.

La représentation $R_{\bar{w}}$ peut être prolongée sur tout $L_{w}$ :

$$
r_{\bar{w}}(a) \psi_{\bar{w}}(b, f)=\psi_{w}(a b, f) .
$$

En ce qui suit nous supposons $R_{\bar{w}}$ définie sur $L_{\bar{w}}$. 
Soit $L_{\infty}(\mu, s)$ l'espace des fonctions $\mu$-bornées [8] sur $s$ muni de la norme:

$$
\|f\|_{L_{\infty}(\mu, s)}=\operatorname{Vrai\operatorname {sup}}|f(w)| .
$$

Soit $f$ une fonction de l'espace $L_{\infty}(\mu, s)$. Considérons l'opérateur $B(f)$ défini sur tout $H_{\bar{w}}$ par l'équation:

$$
[B(f) x]_{w}=f(w)[x]_{w} .
$$

Il est évident que l'opérateur $B(f)$ est borné:

$$
\|B(f)\|_{H_{w}}=\|f\|_{L_{\infty}(\mu, s)}
$$

et appartient au commutant de la représentation $R_{\bar{w}}$.

Proposition $\check{\text {. }}$. Tous les opérateurs de $R_{\bar{w}}^{\prime}$ sont de ce genre:

$$
R_{\bar{w}}^{\prime}=\left\{B(f): f \in L_{\infty}(\mu, s)\right\} .
$$

Soit un $B \in R_{\bar{w}}^{\prime}$. En vertu du lemme $2 B \psi_{\bar{w}}(1,1)$ est un vide dans $H_{\bar{w}}$. Il existe donc une fonction $f \in L_{2}(\mu, s)$ telle que:

Alors:

$$
B \psi_{\bar{w}}(1,1)=\psi_{\bar{w}}(1, f) \text {. }
$$

$$
\begin{aligned}
\left\langle\psi_{\bar{w}}(a, 1), B \psi_{\bar{w}}(b, 1)\right\rangle & =\left\langle\psi_{\bar{w}}\left(b^{+} a\right), B \psi_{\bar{w}}(1,1)\right\rangle \\
& =\left\langle\psi_{\bar{w}}\left(b^{+} a, 1\right), \psi_{\bar{w}}(1, f)\right\rangle=\left\langle\psi_{\bar{w}}(a, 1), \psi_{\bar{w}}(b, f)\right\rangle
\end{aligned}
$$

d'où $B \psi_{\bar{w}}(b, 1)=\psi_{\bar{w}}(b, f)$ pour tout $b \in A$ à la suite de la cyclicité démontrée.

Il nous reste à démontrer que $f \in L_{\infty}(\mu, s)$. B étant borné par hypothèse, l'inégalité:

$$
\begin{aligned}
\left\|B \psi_{\bar{w}}(a, 1)\right\|^{2}=\left\|\psi_{\bar{w}}(a, f)\right\|^{2} & =\int_{s} w\left(a^{+} a\right)|f(w)|^{2} d \mu(w) \\
& \leqq\|B\|^{2}\left\|\psi_{\bar{w}}(a, 1)\right\|^{2}=\|B\|^{2} \int_{s} w\left(a^{+} a\right) d \mu(w)
\end{aligned}
$$

est valide pour tout $a \in A$. Nous désirons démontrer qu'il en résulte:

$$
V \underset{w \in s}{\operatorname{ai} \sup }|f(w)| \leqq\|B\| \text {. }
$$

En effet, dans le cas contraire il existe $\delta>0$ tel que:

$$
\mu\left\{w \in s:|f(w)|^{2} \geqq\|B\|^{2}+\delta\right\} \equiv \mu(e)>0 .
$$

Désignons par $\chi_{e}$ la fonction caractéristique de l'ensemble $e$ :

$$
\chi_{e}(w)= \begin{cases}1 & w \in e \\ 0 & w \notin e .\end{cases}
$$

Evidemment $\chi_{e} \in L_{2}(\mu, s)$ d'où $\psi_{w}\left(1, \chi_{e}\right) \in H_{\bar{w}}$. En vertu de la proposition 3 il existe la suite $a_{n} n=1,2, \ldots$ telle que la suite $\psi_{\bar{w}}\left(a_{n}, 1\right)$ 
converge vers $\psi_{\bar{w}}\left(1, \chi_{e}\right)$ dans la norme de $H_{\bar{w}}$. On a :

$$
\begin{aligned}
\|B\|^{2} \int_{s} w\left(a_{n}^{+} a_{n}\right) d \mu(w)= & \|B\|^{2}\left\|\psi_{\bar{w}}\left(a_{n}, 1\right)\right\|^{2} \\
& \rightarrow\|B\|^{2}\left\|\psi_{\bar{w}}\left(1, \chi_{e}\right)\right\|^{2}=\|B\|^{2} \mu(e)
\end{aligned}
$$

et en même temps:

$$
\begin{aligned}
\int_{s} w\left(a_{n}^{+} a_{n}\right)|f(w)|^{2} d \mu(w)= & \int_{s} w\left(a_{n}^{+} a_{n}\right) \chi_{e}(w)|f(w)|^{2} d \mu(w) \\
& +\int_{s} w\left(a_{n}^{+} a_{n}\right)\left(1-\chi_{e}(w)\right)|f(w)|^{2} d \mu(w) .
\end{aligned}
$$

La deuxième intégrale converge vers 0 . En effet:

$$
\begin{array}{r}
\int_{s} w\left(a_{n}^{+} a_{n}\right)\left(1-\chi_{e}(w)\right)|f(w)|^{2} d \mu(w) \leqq\left[\|B\|^{2}+\delta\right] \int_{s} w\left(a_{n}^{+} a_{n}\right)\left(1-\chi_{e}(w)\right) \\
=\left[\|B\|^{2}+\delta\right]\left\langle\psi_{\bar{w}}\left(a_{n}, 1\right), B\left(1-\chi_{e}\right) \psi_{\bar{w}}\left(a_{n}, 1\right)\right\rangle \\
\rightarrow\left[\|B\|^{2}+\delta\right]\left\langle\psi_{\bar{w}}\left(1, \chi_{e}\right), B\left(1-\chi_{e}\right) \psi_{\bar{w}}\left(1, \chi_{e}\right)\right\rangle=0 .
\end{array}
$$

D'autre part:

$$
\begin{aligned}
\int_{s} w\left(a_{n}^{+} a_{n}\right) \chi_{e}(w)|f(w)|^{2} d \mu(w) \geqq\left[\|B\|^{2}+\delta\right] \int_{s} w\left(a_{n}^{+} a_{n}\right) \chi_{e}(w) d \mu(w) \\
\rightarrow\left[\|B\|^{2}+\delta\right]\left\langle\psi_{\bar{w}}\left(1, \chi_{e}\right), B\left(\chi_{e}\right) \psi_{\bar{w}}\left(1, \chi_{e}\right)\right\rangle=\left[\|B\|^{2}+\delta\right] \mu(e)
\end{aligned}
$$

ce qui contredit l'inégalité initiale. Donc:

$$
V \underset{w \in s}{\operatorname{ain} \sup }|f(w)| \leqq\|B\| \text {. }
$$

Il en résulte que $B(f)$ est borné. Deux opérateurs bornés $B$ et $B(f)$ sont identiques sur $L_{\bar{w}}(1)$, donc $B=B(f)$ partout dans $H_{\bar{w}}$ (cyclicité) ce qui achève la démonstration.

Définition. Nous dirons que deux fonctions $f_{1,2} \in L_{2}(\mu, s)$ sont équivalentes dans $L_{2}(\mu, s)$ [et nous écrirons $f_{1} \sim f_{2} \in L_{2}(\mu, s)$ ] si le sont les mesures $\mu_{1,2}$ déterminées par l'équation:

$$
d \mu_{i}(w) \equiv\left|f_{i}(w)\right|^{2} d \mu(w) \quad i=1,2 .
$$

(Rappelons que les mesures $\mu_{1,2}$ sont dites équivalentes si et seulement si tout ensemble $\mu_{1}$-négligeable est $\mu_{2}$-négligeable et inversement.)

Il résulte de cette définition que si $f_{1} \sim f_{2} \in L_{2}(\mu, s)$ l'une des égalités $\psi_{\bar{w}}\left(a, f_{i}\right)=0 i=1$ ou 2 entraîne l'autre.

Lemme 4. Pour que $L_{\bar{w}}(f)$ soit dense dans $H_{\bar{w}}$ il faut et il suffit que $f$ soit équivalente à 1 dans $L_{2}(\mu, s)$.

La condition est nécessaire. En effet, supposons que $L_{\bar{w}}(f)$ est dense dans $H_{\bar{w}}$ mais $f$ n'est pas équivalente à 1 . Il existe donc un ensemble $e \subset s$ tel que $\mu(e)>0$ et $\mu^{\prime}(e)=0$ où $\mu^{\prime}$ est définie par l'équation $d \mu^{\prime}=|f|^{2} d \mu$. Désignons par $\chi_{e}$ la fonction caractéristique de l'ensemble e. Donc:

$$
\left\|\psi_{\bar{w}}\left(1, \chi_{e}\right)\right\|^{2}=\int_{s} \chi_{e}(w) d \mu(w)>0, \int_{s} \chi_{e}(w)|f(w)|^{2} d \mu(w)=0 .
$$


Il en résulte que le vecteur $\psi_{\bar{w}}\left(1, \chi_{e}\right)$ est orthogonal à $L_{\bar{w}}(f)$ :

$$
\begin{aligned}
\left|\left\langle\psi_{\bar{w}}\left(1, \chi_{e}\right), \psi_{\bar{w}}(a, f)\right\rangle\right|^{2} & =\left|\int_{s} w(a) f(w) \chi_{e}(w) d \mu(w)\right|^{2} \\
& \leqq \int_{s}|w(a)|^{2} d \mu(w) \cdot \int_{s} \chi_{e}(w)|f(w)|^{2} d \mu(w)=0
\end{aligned}
$$

ce qui contredit I'hypothèse.

Démontrons que la condition est suffisante. En vertu de la proposition 3 il suffit de vérifier que $L_{\bar{w}}(f)$ est dense dans $L_{\bar{w}}(1)$ si $f \sim 1 \in L_{2}(\mu, s)$.

Le raisonnement ressemble à la démonstration de la proposition 3 . Soit $\mu^{\prime}$ la mesure définie par l'équation $d \mu^{\prime}=|f|^{2} d \mu$. La fonction $f^{-1}$ appartient évidemment à l'espace $L_{2}\left(\mu^{\prime}, s\right)$. Pour un $a \in A$ fixé, $b \in A$ arbitraire écrivons (voir la démonstration de la proposition 3):

$$
\begin{aligned}
\left\|\psi_{\bar{w}}(a, 1)-\psi_{\bar{w}}\left(a b^{(0)}, f\right)\right\|^{2} & =\int_{s} w\left(a^{+} a\right)\left|1-f_{b}(w) f(w)\right|^{2} d \mu(w) \\
& =\int_{s} w\left(a^{+} a\right)\left|f^{-1}(w)-f_{b}(w)\right|^{2} d \mu^{\prime}(w) \\
& \leqq \operatorname{Sup}_{w \in s} w\left(a^{+} a\right) \int_{s}\left|f^{-1}(w)-f_{b}(w)\right|^{2} d \mu^{\prime}(w) .
\end{aligned}
$$

La dernière intégrale peut être rendue arbitrairement petite par le choix de $b \in A$ car $F(s) \equiv\left\{f_{b}: b \in A\right\}$ étant dense uniformément dans $C_{0}(s)$ est dense dans $L_{2}\left(\mu^{\prime}, s\right)$.

Définition. Nous dirons que deux représentations $R_{\bar{w}_{1}}$ et $R_{\bar{w}_{2}}$ dans $H_{\bar{w}_{1}}$ et $H_{\bar{w}_{2}}$ respectivement sont semblables s'il existe $f_{1} \sim 1 \in L_{2}\left(\mu_{1}, s_{1}\right)$, $f_{2} \sim 1 \in L_{2}\left(\mu_{2}, s_{2}\right)$ et une application linéaire inversible $V$ de $L_{\bar{w}_{1}}\left(f_{1}\right)$ sur $L_{\bar{w}_{2}}\left(f_{2}\right)$ telles que $r_{\bar{w}_{2}}(a)=V r_{\bar{w}_{1}}(a) V^{-1}$ sur $L_{\bar{w}_{2}}\left(f_{2}\right)$. Si $V$ est isométrique, $R_{\bar{w}_{1}}$ et $R_{\bar{w}_{2}}$ seront appelées unitairement équivalentes.

Remarque. D'habitude on définit l'équivalence unitaire en supposant que $R_{\bar{w}_{1}}$ et $R_{\bar{w}_{2}}$ sont définies seulement sur $L_{\bar{w}_{1}}(1)$ et $L_{\bar{w}_{2}}(1)$ respectivement et par suite on doit exiger que $V$ soit l'application de $L_{\bar{w}_{1}}(1)$ sur $L_{\bar{w}_{2}}(1)$.

Dans le reste de ce paragraphe nous formulerons des critères de similitude et d'équivalence unitaire.

Proposition 6. Les deux représentations $R_{\bar{w}_{1}}$ et $R_{\bar{w}_{2}}$ sont unitairement équivalentes si et seulement si $s_{1}=s_{2}$ et $\mu_{1}$ est équivalente $\grave{a} \mu_{2}$.

La condition est suffisante. En effet, soient

$$
\bar{w}_{i}=\int_{s} w d \mu_{i}(w)^{n}, \quad i=1,2, \quad \mu_{1} \sim \mu_{2} .
$$

Il est clair que la fonction $f \equiv\left(\frac{d \mu_{1}}{d \mu_{2}}\right)^{1 / 2}$ (la dérivée de RADON-NICODIME existe en vertu de la condition $\left.\mu_{1} \sim \mu_{2}\right)$ appartient à l'espace $L_{2}\left(\mu_{2}, s\right)$ et "est équivalente à 1 dans $L_{2}\left(\mu_{2}, s\right)$ en vertu de l'équivalence entre $\mu_{1}$ et $\mu_{2}$. L'application linéaire $V$ de $L_{\bar{w}_{1}}(1)$ sur $L_{\bar{w}_{2}}(f)$ définie par l'équation:

$$
V \psi_{\bar{w}_{1}}(a, 1)=\psi_{\bar{w}_{2}}(a, f)
$$

est isométrique et réalise évidemment la similitude en question. 
La condition est aussi nécessaire. En effet supposons qu'il existe $f_{1} \sim 1 \in L_{2}\left(\mu_{1}, s_{1}\right), f_{2} \sim 1 \in L_{2}\left(\mu_{2}, s_{2}\right)$ et un opérateur isométrique $V$ de $L_{\bar{w}_{1}}\left(f_{1}\right)$ sur $L_{\bar{w}_{2}}\left(f_{2}\right)$ tels que $r_{\bar{w}_{2}}(a)=V r_{\bar{w}_{1}}(a) V^{-1}$ sur $L_{\bar{w}_{2}}\left(f_{2}\right)$. En vertu du lemme 4 l'opérateur $V$ peut être prolongé par continuité jusqu'à l'isométrie de $H_{\bar{w}_{1}}$ sur $H_{\bar{w}_{2}}$.

On peut vérifier par un calcul direct que l'opérateur unitaire

$$
V^{-1} U_{\bar{w}_{2}}(\lambda, 1) V U_{\bar{w}_{1}}^{-1}(\lambda, 1)
$$

dans $H_{\bar{w}_{1}}$ appartient au commutant $R_{\bar{w}_{1}}^{\prime}$. Il existe donc une famille de fonctions $h_{\lambda} \in L_{\infty}\left(\mu_{1}, s_{1}\right),\left|h_{\lambda}\right|=1$ (l'unitarité) telle que:

d'où

$$
V^{-1} U_{\bar{w}_{2}}(\lambda, 1) V U_{\bar{w}_{1}}^{-1}(\lambda, 1)=B\left(h_{\lambda}\right)
$$

$$
U_{\bar{w}_{1}}(\lambda, 1)=B\left(h_{\lambda}^{*}\right) V^{-1} U_{\bar{w}_{2}}(\lambda, 1) V .
$$

Nous sommes maintenant en mesure de démontrer que l'opérateur $V$ transforme «le vide en vide». Par exemple, démontrons que le vecteur $V^{-1} \psi_{\bar{w}_{2}}\left(1, f_{2}\right)$ est un vide dans $H_{w_{1}}$.

On a :

$$
U_{\bar{w}_{1}}(\lambda, 1) V^{-1} \psi_{\bar{w}_{2}}\left(1, f_{2}\right)=B\left(h_{\lambda}^{*}\right) V^{-1} \psi_{\bar{w}_{2}}\left(1, f_{2}\right) .
$$

En passant aux composants dans $H_{w}, w \in s_{1}$ :

$$
U_{w}(\lambda, 1)\left[V^{-1} \psi_{\bar{w}_{2}}\left(1, f_{2}\right)\right]_{w}=h_{\lambda}^{*}(w)\left[V^{-1} \psi_{\bar{w}_{2}}\left(1, f_{2}\right)\right]_{w}
$$

voyons que $\left[V^{-1} \psi_{\bar{w}_{2}}\left(1, f_{2}\right)\right]_{w}$ est un vecteur propre de $U_{w}(\lambda, 1)$ dans $H_{w}$. Mais dans $H_{w}$ il n'y a qu'un vecteur propre de $U_{w}(\lambda, 1)$ - c'est le vide $\psi_{w}(1)$, car tous les autres vecteurs de $H_{w}$ appartiennent au spectre continu de l'opérateur d'énergie-impulsion, et par suite $h_{\lambda}(w)=1 \forall w \in s_{1}$ d'où la conclusion.

Une autre démonstration de cette assertion (indépendante) sera donnée au cours de la démonstration de la proposition suivante.

$\mathrm{Il}$ existe donc une fonction $\varphi \in L_{2}\left(\mu_{1}, s_{1}\right)$ telle que:

Il vient:

$$
V^{-1} \psi_{\bar{w}_{2}}\left(1, f_{2}\right)=\psi_{\bar{w}_{1}}(1, \varphi) \text {. }
$$

$$
\begin{aligned}
& \left\langle\psi_{\bar{w}_{2}}\left(1, f_{2}\right), r_{w_{2}}(a) \psi_{\bar{w}_{2}}\left(1, f_{2}\right)\right\rangle=\int_{s_{2}} w(a)\left|f_{2}(w)\right|^{2} d \mu_{2}(w) \\
& \quad=\left\langle V^{-1} \psi_{\bar{w}_{2}}\left(1, f_{2}\right), r_{\bar{w}_{1}}(a) V^{-1} \psi_{\bar{w}_{2}}\left(1, f_{2}\right)\right\rangle=\int_{s_{1}} w(a)|\varphi(w)|^{2} d \mu_{1}(w) .
\end{aligned}
$$

En utilisant l'unicité d'une telle représentation démontrée à la fin du premier paragraphe nous concluons que $s_{2} \leqq s_{1}$ et $d \mu_{2}^{\prime} \equiv\left|f_{2}\right|^{2} d \mu_{2}=|\varphi|^{2} d \mu_{1}$ d'où $\mu_{2}^{\prime} \ll \mu_{1}\left(\mu_{2}^{\prime}\right.$ est absolument continue par rapport à $\left.\mu_{1}\right)$, donc $\mu_{2} \ll \mu_{1}$ en vertu de l'équivalence entre $f_{2}$ et 1 .

En permutant $\bar{w}_{1}$ et $\bar{w}_{2}$ dans le raisonnement nous pouvons démontrer l'inverse: $s_{1} \leqq s_{2}, \mu_{1} \ll \mu_{2}$, d'où la proposition. 
Cette démonstration ne s'etend pas au cas général de la similitude car bien que l'opérateur $V^{-1} U_{\bar{w}_{2}}(\lambda, 1) V U_{\bar{w}_{1}}^{-1}(\lambda, 1)$ commute formellement avec $R_{\bar{w}_{1}}$ on ne peut pas affirmer qu'il appartient au commutant $R_{\bar{w}_{1}}^{\prime}$ sans avoir vérifié qu'il est borné.

On peut facilement formuler la condition suffisante de similitude: deux représentations $R_{\bar{w}_{1}}$ et $R_{\bar{w}_{2}}$ sont semblables si

$$
\Omega\left(\bar{w}_{1}\right) \equiv\left\{a \in A: \bar{w}_{1}\left(a^{+} a\right)=0\right\}=\left\{a \in A: \bar{w}_{2}\left(a^{+} a\right)=0\right\} \equiv \Omega\left(\bar{w}_{2}\right) .
$$

En effet dans ce cas l'application linéaire $V$ de $L_{\bar{w}_{2}}(1)$ sur $L_{w_{2}}(1)$ définie par l'équation:

$$
V \psi_{\bar{w}_{1}}(a, 1)=\psi_{\bar{w}_{2}}(a, 1)
$$

est inversible et réalise évidemment la similitude.

Proposition 7. L'égalité $\Omega\left(\bar{w}_{1}\right)=\Omega\left(\bar{w}_{2}\right)$ est la condition nécessaire et suffisante de similitude entre $R_{\bar{w}_{1}}$ et $R_{\bar{w}_{2}}$.

Il nous reste à démontrer que la condition est nécessaire. Soient donc $f_{1} \sim 1 \in L_{2}\left(\mu_{1}, s_{1}\right), \quad f_{2} \sim 1 \in L_{2}\left(\mu_{2}, s_{2}\right)$, l'application inversible $V$ de $L_{\bar{w}_{1}}\left(f_{1}\right)$ sur $L_{\bar{w}_{2}}\left(f_{2}\right)$ telles que $r_{\bar{w}_{2}}(a)=V r_{\bar{w}_{1}}(a) V^{-1}$ sur $L_{\bar{w}_{2}}\left(f_{2}\right)$. Démontrons que $V$ transforme le vide dans $H_{\bar{w}_{1}}$ en vide dans $H_{\bar{w}_{2}}$.

Par définition il existe $a_{0} \in A$ tel que:

et

$$
V \psi_{\bar{w}_{1}}\left(1, f_{1}\right)=\psi_{\bar{w}_{2}}\left(a_{0}, f_{2}\right)
$$

$$
V \psi_{\bar{w}_{1}}\left(a, f_{1}\right)=\psi_{\bar{w}_{2}}\left(a a_{0}, f_{2}\right) \quad \forall a \in A .
$$

Décomposons $a_{0}$ comme d'habitude:

$$
a_{0}=a_{0}^{(0)}+a_{0}^{(1)}+a_{0}^{(2)} .
$$

En vertu de la condition spectrale forte:

$$
\psi_{\bar{w}_{2}}\left(a_{0}, f_{2}\right)=\psi_{\bar{w}_{2}}\left(a_{0}^{(0)}, f_{2}\right)+\psi_{\bar{w}_{2}}\left(a_{0}^{(1)}, f_{2}\right) .
$$

Il nous faut done montrer que $\psi_{\bar{w}_{2}}\left(a_{0}^{(1)}, f_{2}\right)=0$.

Soit $a \in A$ un élément tel que $\operatorname{Supp}_{p} a$ soit contenu dans la région $p_{0} \leqq-\varepsilon<0$. En vertu de la condition spectrale $\psi_{\bar{w}_{1}}\left(a, f_{1}\right)=0$ d'où ( $V$ est biunivoque par condition):

$$
\psi_{\bar{w}_{2}}\left(a a_{0}^{(0)}, f_{2}\right)+\psi_{\bar{w}_{2}}\left(a a_{0}^{(1)}, f_{2}\right)=0 .
$$

Le premier membre est nul en vertu de la condition spectrale, donc:

$$
\psi_{\overline{n^{2}}}\left(a a_{0}^{(1)}, f_{2}\right)=0
$$

ce qui est valide pour tout $a \in A$ de ce genre. Il vient:

$$
\left\langle\psi_{\bar{w}_{2}}\left(1, f_{2}\right), \psi_{\bar{w}_{2}}\left(a a_{0}^{(1)}, f_{2}\right)\right\rangle=\left\langle\psi_{\bar{w}_{2}}\left(a^{+}, f_{2}\right), \psi_{\bar{w}_{2}}\left(a_{0}^{(1)}, f_{2}\right)\right\rangle=0 .
$$

Puisque $\operatorname{Supp}_{p} a^{+}=-\operatorname{Supp}_{p} a$ nous voyons que $\psi_{\bar{w}_{2}}\left(a_{0}^{(1)}, f_{2}\right)$ est orthogonal à tous les vecteurs de la forme $\psi_{\bar{w}_{2}}\left(a, f_{2}\right)$ où $a$ est tel que Supp $\mathrm{Su}_{p} a$ est contenu dans la région $p_{0} \geqq \varepsilon>0$ et comme il est orthogonal à tout 
vide dans $H_{\bar{w}_{2}}$ par construction nous concluons qu'il est orthogonal à tout vecteur de $L_{\bar{w}_{2}}\left(f_{2}\right)$. En appliquant le lemme 4 nous concluons que $\psi_{\overline{w_{2}}}\left(a_{0}^{(1)}, f_{2}\right)$ est nul.

Il existe donc une fonction $h \in L_{2}\left(\mu_{2}, s_{2}\right)$ telle que:

et

$$
V \psi_{\bar{w}_{1}}\left(1, f_{1}\right)=\psi_{\bar{w}_{2}}(1, h)
$$

pour tout $a \in A$.

$$
V \psi_{\bar{w}_{1}}\left(a, f_{1}\right)=\psi_{\bar{w}_{2}}(a, h)
$$

Soit un $a \in \dot{\Omega}\left(\bar{w}_{2}\right)$. On a $\psi_{\bar{w}_{2}}(a, h)=0$ d'où $\psi_{\bar{w}_{1}}\left(a, f_{1}\right)=0$ ( $V$ est biunivoque par hypothèse) et par suite $\psi_{\bar{w}_{2}}(a, 1)=0$ donc $a \in \Omega\left(\bar{w}_{1}\right)$. Cela signifie que $\Omega\left(\bar{w}_{2}\right) \leqq \Omega\left(\bar{w}_{1}\right)$. En permutant $\bar{w}_{1}$ et $\bar{w}_{2}$ dans le raisonnement nous obtenons l'inclusion inverse d'où la proposition.

Remarque. On pourrait s'imaginer qu'en écrivant:

$$
\left\langle\psi_{\bar{w}_{1}}\left(1, f_{1}\right), r_{\bar{w}_{1}}(a) \psi_{\bar{w}_{1}}\left(1, f_{1}\right)\right\rangle=\left\langle\left(V^{-1}\right)^{+} \psi_{\bar{w}_{1}}\left(1, f_{1}\right), r_{\bar{w}_{2}}(a) V \psi_{\bar{w}_{1}}\left(1, f_{1}\right)\right\rangle
$$

et on essayant de démontrer par analogie que $\left(V^{-1}\right)^{+} \psi_{\bar{w}_{1}}\left(1, f_{1}\right)$ est un vide dans $H_{\bar{w}_{2}}$ on arrivera à la conclusion que toute similitude est l'équivalence unitaire. Mais cette conclusion est fausse ce que nous démontrerons par un contre-exemple à la fin de ce paragraphe. On n'a pas le droit de supposer $\left(V^{-1}\right)^{+}$toujours défini sur $\psi_{\bar{w}_{1}}\left(1, f_{1}\right)$.

Etant fausse en générale cette conclusion est vraie lorsque $s$ est fini (c'est à dire lorsque $s$ contient un nombre fini de points). Cette assertion a été énoncée dans l'article [9] comme générale, mais la démonstration proposée n'est valide que pour des fonctionelles de la classe $Q$.

Proposition 8. Soit une $\bar{w} \in Q$ telle que s est fini:

$$
\bar{w}=\sum_{i=1}^{n} \varrho_{i} w_{i}, \quad \varrho_{i}>0 \sum_{i=1}^{n} \varrho_{i}=1, \quad w_{i} \in Q_{0}, \quad i=1 \ldots n .
$$

T'oute $w^{\prime} \in K_{0}$ telle que $\Omega\left(w^{\prime}\right) \geqq \Omega(\bar{w})$ est composée des mémes fonctionelles:

$$
w^{\prime}=\sum_{i=1}^{n} \varrho_{i}^{\prime} w_{i}, \quad \varrho_{i}^{\prime} \geqq 0 \sum_{i=1}^{n} \varrho_{i}^{\prime}=1
$$

Les coefficients $\varrho_{i}^{\prime}$ sont strictement positifs si et seulement si $\Omega\left(w^{\prime}\right)=\Omega(\bar{w})$.

Démonstration. Soient $R_{\bar{w}}, R_{w^{\prime}}$ les représentations dans $H_{\bar{w}}, H_{w^{\prime}}$ respectivement. L'application $V$ de $L_{\bar{i}}(1)$ sur $L_{w^{\prime}}$ :

$$
V \psi_{\bar{w}}(a, 1)=\psi_{w^{\prime}}(a)
$$

est univoque par condition (et biunivoque si $\Omega\left(w^{\prime}\right)=\Omega(\bar{w})$ ). Ecrivons comme d'habitude:

Alors:

$$
a=a^{(0)}+a^{(1)}+a^{(2)} .
$$

$$
w^{\prime}(a)=w^{\prime}\left(a^{(0)}\right)=\left\langle\psi_{w^{\prime}}(1), \psi_{w^{\prime}}\left(a^{(0)}\right)\right\rangle=\left\langle V \psi_{\bar{w}}(1,1), V \psi_{\bar{w}}\left(a^{(0)}, 1\right)\right\rangle .
$$


Le vecteur $\psi_{\bar{w}}\left(a^{(0)}, 1\right)$ est un vide dans $H_{\bar{w}}$ dont les composants dans $H_{w_{i}}$ sont:

$$
\psi_{w_{i}}\left(a^{(0)}\right)=w_{i}\left(a^{(0)}\right) \psi_{w_{i}}(1)=w_{i}(a) \psi_{w_{i}}(1), \quad i=1 \ldots n .
$$

Le probléme est donc réduit à l'analyse de l'application $V$ sur l'espace de dimension finie $H_{\bar{w}}^{0} \subset H_{\bar{w}}$ dont la base est formée par les vides $\psi_{w_{i}}(1)$ $i=1 \ldots n$.

L'ensemble $\psi_{\bar{w}}\left(a^{(0)}, 1\right): a \in A$ est dense dans $H_{\bar{w}}^{0}$ (c'est une simple conséquence du lemme 3 ), donc, il coîncide avec $H \bar{w}$, ce qui signifie que $V$ est définie sur tout $H_{\tilde{w}}^{0}$. On a:

$$
w^{\prime}(a)=\sum_{i, k=1}^{n} w_{i}(1) w_{k}(a)\left\langle V \psi_{w_{i}}(1), V \psi_{w_{k}}(1)\right\rangle \equiv \sum_{i=1}^{n} \varrho_{i}^{\prime} w_{i}(a) .
$$

Démontrons, que les coefficients $\varrho_{i}^{\prime} i=1 \ldots n$ sont positifs. En effet, l'égalité:

implique:

$$
\begin{aligned}
w^{\prime}\left(a^{(0)+} a^{(0)}\right) & =\sum_{i, k=1}^{n} w_{i}(a)^{*} w_{k}(a)\left\langle V \psi_{w_{i}}(1), V \psi_{w_{k}}(1)\right\rangle \\
& =\sum_{i=1}^{n} \varrho_{i}^{\prime} w_{i}\left(a^{(0)+} a^{(0)}\right)=\sum_{i=1}^{n} \varrho_{i}^{\prime}\left|w_{i}(a)\right|^{2}
\end{aligned}
$$

d'où la conclusion.

$$
\left\langle V \psi_{w_{i}}(1), V \psi_{w_{k}}(1)\right\rangle=\delta_{i k} \varrho_{i}^{\prime}
$$

Il nous reste à démontrer la dernière assertion de la proposition. Il est clair que la positivité stricte des coefficients $\varrho_{i}^{\prime}$ entraîne l'égalité $\Omega\left(w^{\prime}\right)=\Omega(\bar{w})$. Inversement, si $\Omega\left(w^{\prime}\right)=\Omega(\bar{w})$ en permutant $\bar{w}$ et $w^{\prime}$ dans le raisonnement (ce qui est possible parce qu'on a déjà démontré que $w^{\prime} \in Q$ ) nous arrivons à la conclusion que $\bar{w}$ est composée des mémes fonctionelles que $w^{\prime}$, ce qui n'est possible qu'en cas où tous les coefficients $\varrho_{i}^{\prime} i=1 \ldots n$ sont strictement positifs.

Il est clair que dans le dernier cas l'ensemble des coefficients $\varrho_{i}^{\prime}>0$ $i=1 \ldots n$ définit sur $s \equiv \bigcup_{i=1}^{n} w_{i}$ une mesure équivalente à la mesure initiale et les représentations $R_{\bar{w}}$ et $R_{w^{\prime}}$ sont unitairement équivalentes en vertu de la proposition 5 .

Cette assertion ne subsiste pas lorsque la dégénération du vide est infinie. Nous allons construire un contre-exemple.

Soit $w_{0} \in Q_{0}$ une fonctionelle engendrée par un champ complexe $A_{i}(x) i=1,2$ où :

$$
A_{1}(x) \equiv A(x), \quad A_{2}(x) \equiv A^{+}(x) .
$$

Soit $G \equiv\left\{g(\alpha): \alpha \in R^{1}\right\}$ le groupe des * automorphismes représentant les transformations de jauge du champ $A_{i}(x)$ :

où $i_{\alpha}=1,2, \varepsilon_{1}=-1, \varepsilon_{2}=1$.

$$
(g(\alpha) a)_{i_{1} \ldots i_{n}}\left(x_{1} \ldots x_{n}\right)=a_{i_{1} \ldots i_{n}}\left(x_{1} \ldots x_{n}\right) \exp i \alpha \sum_{k=1}^{n} \varepsilon_{i_{k}}
$$


Supposons que $w_{0}$ n'est pas invariante par le groupe $G$ :

$$
w_{\alpha}(a) \equiv w_{0}(g(\alpha) a) \neq w_{0}(a) .
$$

En vertu de la proposition 2 toutes les $w_{\alpha}: \alpha \in R^{1}$ appartiennent à la classe $Q_{0}$. On peut aussi affirmer que la borne inférieure du spectre continu de l'opérateur d'énergie-impulsion est la même pour toute fonctionelle $w_{\alpha}: \alpha \in R^{1}$ (voir la démonstration de la proposition 2 ). Il en résulte que toute partie de l'ensemble $s\left(R^{1}\right) \equiv\left\{w_{\alpha}: \alpha \in R^{1}\right\}$ satisfait automatiquement à la condition 5 du paragraphe précédent.

De telles fonctionelles existent. L'exemple le plus simple d'une telle fonctionelle est celle engendrée par le champ

$$
\begin{aligned}
& A_{1}(x)=A_{m}(x)+c \\
& A_{2}(x)=A_{m}^{+}(x)+c^{*}
\end{aligned}
$$

où $A_{m}(x)$ est le chambre complexe libre de la masse $m, c \neq 0$ est un nombre réel ou complexe.

Il est évident que cette fonctionelle appartient à la classe $Q_{0}$ (car la représentation unitaire du groupe de PoINCARÉ est la même que pour le champ complexe libre) et n'est pas invariante par le groupe $G$. Il est également évident que cet exemple n'est pas unique dans la classe de Borchers du champ complexe libre.

Soit donc une telle fonctionelle. Trouvons les conditions auxquelles doit satisfaire un ensemble $e$ de points de l'axe réel $R^{1}$ pour que l'ensemble des fonctionelles $s(e) \equiv\left\{w_{\alpha}: \alpha \in e\right\}$ satisfasse aux conditions 4,5 du premier paragraphe.

Nous avons démontré ci-dessus que $s(e)$ satisfait toujours à la condition 5 . Il est aussi évident que $s(e)$ est borné pour tout ensemble $e<R^{1}$. La restriction non-triviale peut provenir seulement de la condition que $s(e)$ soit faiblement fermé.

Il est clair que pour que l'ensemble $s(e)$ soit faiblement fermé il faut et il suffit que le soit l'ensemble des distributions $w_{i_{1}}^{(\alpha)} \ldots i_{n}\left(x_{1} \ldots x_{n}\right): \alpha \in e$ pour tout ensemble d'indices $i_{1} \ldots i_{n}$. fixé.

La fonction $w_{i_{1} \ldots i_{n}}^{(\alpha)}\left(x_{1} \ldots x_{n}\right)$ dépend de $\alpha$ par l'intermédiaire du facteur de la forme $\exp i \alpha N\left(i_{1} \ldots i_{n}\right)$ où $N\left(i_{1} \ldots i_{n}\right)$ est le nombre de champs $A_{2}$ moins celui de champs $A_{1}$ contenus dans cette fonction. En vertu du lemme 3 l'ensemble des fonctions $\exp i \alpha N\left(i_{1} \ldots i_{n}\right): w_{i_{1} \ldots i_{n}}^{(0)} \neq 0$ contient avec deux fonctions $\exp i n \alpha, \exp i n^{\prime} \alpha$ leur produit $\exp i\left(n+n^{\prime}\right) \alpha$ et leurs conjuguées $\exp (-i n \alpha)$ et $\exp \left(-i n^{\prime} \alpha\right)$. Autrement dit, ces fonctions constituent un groupe. Il en résulte qu'il existe un entier $m>0$ tel que:

$\left\{\exp i \alpha N\left(i_{1} \ldots i_{n}\right): w_{i_{1}}^{(0)} \ldots i_{n} \neq 0\right\}=\{\exp i m n \alpha: n=0, \pm 1, \pm 2, \ldots\}$. 7 Commun. math. Phys., vol. 13 
On déduit done le critère suivant: pour que l'ensemble $s(e)$ soit faiblement fermé il faut et il suffit que le soit l'ensemble des points $\{\exp i m \alpha: \alpha \in e\}$. La condition que $e \subset R^{1}$ soit fermé est suffisante mais elle n'est pas nécessaire.

Soit $e \subset R^{1}$ un ensemble de points de l'axe réel tel que $s(e)$ soit infini (il revient au même de dire que l'ensemble des points $\exp i m \alpha: \alpha \in e$ soit infini). Démontrons que

En effet soit

$$
\bigcap_{\alpha \in e} \Omega\left(w_{\alpha}\right)=\bigcap_{\alpha \in R^{1}} \Omega\left(w_{\alpha}\right) .
$$

$$
a \in \bigcap_{\alpha \in e} \Omega\left(w_{\alpha}\right) .
$$

Cela signifie que $w_{\alpha}\left(a^{+} a\right)=0 \quad \forall \alpha \in e$. Etant considérée comme fonction de $\alpha w_{\alpha}\left(a^{+} a\right)$ est holomorphe et périodique, d'où la conclusion.

Soit un $\alpha^{\prime} \in R^{1}$ tel que $w_{\alpha^{\prime}} \notin s(e)$. On a:

$$
\Omega\left(w_{\alpha^{\prime}}\right) \geqq \bigcap_{\alpha \in e} \Omega\left(w_{\alpha}\right)=\bigcap_{\alpha \in R^{1}} \Omega\left(w_{\alpha}\right) .
$$

Quand même on ne peut pas représenter $w_{\alpha^{\prime}}$ par une intégrale sur $s(e)$ parce que $w_{\alpha^{\prime}}$ est extrémale. Cet exemple montre que la conclusion de la proposition 7 ne subsiste pas lorsque $s$ est infini même s'il est dénombrable.

Soient $e_{\mathbf{1}, 2}$ deux ensembles de $R^{1}$ tels que $s\left(e_{1,2}\right)$ sont fermés et infinis tous les deux. Les représentations $R_{\bar{v}_{1,2}}$ engendrées par les fonctionelles $\bar{w}_{1,2}$

$$
\bar{w}_{i}=\int_{s\left(e_{i}\right)} w_{\alpha} d \mu_{i}\left(w_{\alpha}\right) \quad i=1,2 \quad \operatorname{Supp} \mu_{i}=s\left(e_{i}\right)
$$

sont semblables car d'après la démonstration ci-dessus:

$$
\Omega\left(\bar{w}_{1}\right)=\bigcap_{\alpha \in e_{1}} \Omega\left(w_{\alpha}\right)=\bigcap_{\alpha \in R^{1}} \Omega\left(w_{\alpha}\right)=\bigcap_{\alpha \in e_{2}} \Omega\left(w_{\alpha}\right)=\Omega\left(\bar{w}_{2}\right) .
$$

Mais ces représentations ne sont pas unitairement équivalentes si $s\left(e_{1}\right) \neq s\left(e_{2}\right)$ en vertu de la proposition 5 .

\section{Le problème de la symétrie pour les fonctionelles de la classe $\boldsymbol{Q}$}

Soient $T$ le groupe de tous les *-automorphismes continus de l'algèbre $A, T^{10}$ son sous-groupe d'automorphismes permutables avec les *automorphismes du groupe de Poincaré, une fonctionelle $\bar{w} \in Q, R_{\bar{w}}$ la représentation correspondante dans $H_{\bar{w}}$. Désignons:

$T_{1}(\bar{w}) \equiv\left\{\tau \in T: \bar{w}_{\tau}=\bar{w}\right\}$ le groupe de symétrie de la fonctionelle $\bar{w}$,

$T_{2}(\bar{w}) \equiv\left\{\tau \in T: R_{\bar{w}_{\tau}}\right.$ est unitairement équivalente à $\left.R_{\bar{w}}\right\}$,

$T_{3}(\bar{w}) \equiv\left\{\tau \in T: R_{\bar{w}_{\tau}}\right.$ est semblable à $\left.R_{\bar{w}}\right\}$,

$T_{4}(\bar{w}) \equiv\left\{\tau \in T: R_{\bar{w}_{\tau}}\right.$ est isomorphe à $\left.R_{\bar{w}}\right\}$.

Rappelons que $R_{w_{1}}$ et $R_{w_{2}}$ sont appelées isomorphes si et seulement si la correspondance $r_{w_{1}}(a) \sim r_{w_{2}}(a)$ est biunivoque. 
Par définition:

$$
T_{1}(\bar{w}) \leqq T_{2}(\bar{w}) \leqq T_{3}(\bar{w}) \leqq T_{4}(\bar{w})
$$

pour toute fonctionelle $\bar{w} \in Q$. Désignons:

$$
T_{i}^{0}(\bar{w}) \equiv T_{i}(\bar{w}) \cap T^{0} \quad i=1,2,3,4 .
$$

Soient $\bar{w} \in Q, \tau \in T, R_{\bar{w}}$ et $R_{\bar{w}_{\tau}}$ les représentations dans $H_{\bar{w}}$ et $H_{\bar{w}_{\tau}}$ respectivement (nous n'identifions pas $H_{\bar{v},}$ et $H_{\bar{w}_{\tau}}$ ). L'application $V\left(\bar{w}_{\tau}, \bar{w}\right)$ de $L_{\bar{w}}(1) \operatorname{sur} \mathrm{L}_{\bar{w}_{\tau}}(1)$

$$
V\left(\bar{w}_{\tau}, \bar{w}\right) \psi_{\bar{w}}(\tau a, 1)=\psi_{\bar{w}_{\tau}}(a, 1) \quad \forall a \in A
$$

est évidemment isométrique. En utilisant les définitions, nous obtenons:

$$
r_{\bar{w}_{\tau}}(a)=V\left(\bar{w}_{\tau}, \bar{w}\right) r_{\bar{w}}(\tau a) V^{-1}\left(\bar{w}_{\tau}, \bar{w}\right) .
$$

Si on identifie $H_{-\tau}$ à $H_{\bar{w}}$ par l'isométrie $V^{-1}\left(\bar{w}_{\tau}, \bar{w}\right)$, on arrive aux relations:

$$
\psi\left(\bar{w}_{\tau} 1,1\right)=\psi_{\bar{w}}(1,1) \quad r_{\bar{w}_{\tau}}(a)=r_{\bar{w}}(\tau a)
$$

C'est la réalisation canonique de la représentation transformée par un *-automorphisme $\tau \in T$.

Les propositions démontrées dans le paragraphe précédent rendent évidentes les assertions ci-dessous:

Proposition 9. Pour toute $w \in Q_{0} T_{1}^{0}(w)=T_{2}^{0}(w)=T_{3}^{0}(w)$.

Proposition 10. Soit une $\bar{w} \in Q$. Pour qu'un groupe $T \subset T^{0}$ appartienne $\grave{a}$ $T_{3}^{0}(\bar{w})$ il faut et il suffit que l'ensemble $\Omega(\bar{w})$ soit T-invariant:

$$
\tau \Omega(\bar{w})=\Omega(\bar{w}) \quad \forall \tau \in T .
$$

Proposition 11. Pour toute $\bar{w} \in Q$ telle que s est fini

$$
T_{2}^{0}(\bar{w})=T_{3}^{0}(\bar{w}) .
$$

Proposition 12. Soit une $\bar{w} \in Q$. Pour qu'un groupe $T \subset T^{0}$ appartienne $\grave{a} T_{2}^{0}(\bar{w})$ il faut et il suffit que:

1. $s$ soit T-invariant:

$$
\tau s=s \quad \forall \tau \in T .
$$

2. la mesure $\mu$ sur $s$ soit $T$-quasi-invariante.

(La mesure $\mu$ sur $s$ est appelée $T$-quasi-invariante si la mesure $\tau$-transformée: $d \mu_{\tau}(w) \equiv d \mu\left(w_{\tau}\right)$ est équivalente à la mesure initiale pour tout $\tau \in T$.)

Soit un groupe $T \subset T_{2}^{0}(\bar{w})$. Il est facile à vérifier que l'ensemble d'opérateurs $U(\tau): \tau \in T$ dans $H_{\bar{w}}$ définis par l'équation:

$$
[U(\tau) x]_{w}=V\left(w, w_{\tau}\right)[x]_{w_{\tau}}\left(\frac{d \mu\left(w_{\tau}\right)}{d \mu(w)}\right)^{1 / 2} \quad \forall x \in H_{\bar{w}}
$$

où $V\left(w, w_{\tau}\right)$ - l'isométrie de $H_{w_{\tau}}$ sur $H_{w}$ :

$$
V\left(w, w_{\tau}\right) \psi_{w_{\tau}}(a)=\psi_{w}(\tau a) \quad \forall a \in A
$$


réalise une représentation unitaire du groupe $T$ dans $H_{\bar{w}}$ :

$$
U^{+}(\tau)=U^{-1}(\tau) \quad U(\tau) r_{\bar{w}}(a) U^{-1}(\tau)=r_{\bar{w}}(\tau a) \equiv r_{\bar{w}_{\tau}}(a) .
$$

S'il existe dans $s$ des fonctionelles $T$-invariantes, cette réalisation est essentiellement non-unique, car on peut aussi construire la réalisation normale :

$$
\widetilde{U}(\tau)=\int_{s} \oplus\left[U_{w}(\tau) \chi(w)+1 \cdot(1-\chi(w))\right]
$$

où $\chi(w)$ est la fonction caractéristique de l'ensemble des fonctionelles $T$-invariantes, $U_{w}(\tau)$ la représentation unitaire de $T$ dans $H_{w}$ construite par le procédé du théorème de la reconstruction à partir d'une fonctionelle $w \in s$ qui est $T$-invariante.

La dernière réalisation est non-triviale dans le sous-espace

$$
\tilde{H}_{\bar{w}}=\int_{s} \oplus H_{w} \chi(w) d \mu(w) .
$$

Nous n'avons rien dit sur $T_{4}^{0}(\bar{w})$ car nous ne connaissons pas de critère non-trivial de l'isomorphisme. Enonçons quand même une assertion triviale: pour qu'un groupe $T \subset T^{0}$ appartienne à $T_{4}^{0}(\bar{w})$ il faut et il suffit que le noyau de la représentation $M\left(R_{\bar{w}}\right) \equiv\left\{a \in A: r_{\bar{w}}(a)=0\right\}$ soit $T$-invariant.

En conclusion revenons à l'exemple considéré à la fin du paragraphe précédent et étudions la symétrie des représentations réductibles:

1. $\bar{w}=\sum_{i=1}^{n} \varrho_{i} w_{\alpha_{i}}$ (s est fini). Alors $G \varangle T_{3}^{0}(\bar{w})$ en vertu des propositions 11,12 .

2. $\bar{w}=\sum_{i=1}^{\infty} \varrho_{i} w_{\alpha_{i}}$ (s est dénombrable). Alors $G \subset T_{3}^{0}(\bar{w})$ parce que

$$
\Omega(\bar{w})=\bigcap_{i=1}^{\infty} \Omega\left(w_{\alpha_{i}}\right)=\bigcap_{\alpha \in R^{1}} \Omega\left(w_{\alpha}\right)
$$

est $G$-invariant, mais $G \varangle T_{2}^{0}(\bar{w})$ car $s \equiv \bigcup_{i=1}^{\infty} w_{\alpha_{i}}$ n'est pas $G$-invariant (proposition 5).

3. $\bar{w}=\int_{0}^{2 \pi / m} w_{\alpha} d \mu(\alpha)$ où $2 \pi / m$ est la période maximale (voir la description des fonctionelles $w_{\alpha}$ ). Alors: si $\mu$ n'est pas $G$-quasi-invariante (les mesures $G$-quasi-invariantes sont toutes équivalentes à celle de LebesGue) $G \subset T_{3}^{0}(\bar{w}), G \varangle T_{2}^{0}(\bar{w})$, si $\mu$ est $G$-quasi-invariante mais non invariante, $G \subset T_{2}^{0}(\bar{w}), G \varangle T_{1}^{0}(\bar{w})$, et enfin si $\mu$ est $G$-invariante:

le groupe $G$ appartient à $T_{1}^{0}(\bar{w})$.

$$
\bar{w}=\frac{m}{2 \pi} \int_{0}^{2 \pi i m} w_{\alpha} d \alpha
$$

Cet exemple montre clairement le rapport entre la dégénération du vide et la symétrie. 


\title{
References
}

1. Wightman, A. S.: Phys. Rev. 101, 860 (1956).

2. Borchers, H. J.: Nuovo Cimento 24, 214 (1962).

3. Gelfand, I. M., and N. Ya. Vilenkin: Generalized functions. vol. 4, chap. 1. New York: Academic Press Inc. 1964.

4. Ruelle, D.: Helv. Phys. Acta 35, 1 (1962); 35, 162 (1962).

5. Bourbakr, N.: Eléments de mathématique, Esp. vect. top., chap. 4, § 2 .

6. [5], Top. gén., chap. 10, $2^{e}$ éd. $§ 4$.

7. [5], Intégration, chap. $4,2^{e}$ éd. $\S 2$.

8. [5], Intégration, chap. $4,2^{e}$ éd. $\$ 6$.

9. VAssilev, A. N.: Soviet Phys. JETP 22, 769 (1966).

\author{
A. N. VASSILEV \\ Leningrad State University \\ Department of Theoretical Physics \\ Leningrad, B-164, USSR
}

